\title{
Analysis into Proximity-Coupled Microstrip Antenna on Dielectric Lens
}

\author{
Lawrence Mall \\ Mall Science and Engineering Research \\ Australia
}

\section{Introduction}

Analysis into proximity-coupled microstrip antenna on dielectric lens for characteristics with high gain and pencil beam is presented. Earlier to this work, the University of Michigan developed double-slot antennas on a silicon material lens (Filipovic et al., 1993) and the Swiss Federal Institute of Technology developed aperture-coupled patch antenna on a substrate lens (Eleftheriades et al., 1997). In order to achieve the stringent requirements for efficiency and low cost necessary for a practical radio communication system, we propose our lens antenna for which lens is made of polyethylene plastic for commercial applications. Since the objective is to result in an efficient dielectric lens antenna system, substrates for the proximity-coupled microstrip antenna and the material for the dielectric lens are chosen that they have the same dielectric constant so as to eliminate surface waves and thereof no surface-wave power losses attributed to these selections. We have shown theoretical investigation for how the lens' geometry comes up with dielectric ellipsoid lens and subsequently modified to extended hemispherical dielectric lens as synthesized ellipsoid. Determination of antenna return loss is carried out using method of moments (MoM) which involves spectral domain Green's functions for a proximity-coupled microstrip antenna residing in homogeneous dielectric half space. The Sommerfeld type double integrals are numerically solved and obtained MoM impedance and excitation voltage matrix elements. After solving MoM matrix equation, current column matrix is resulted in and one of which elements is the equivalent current reflection coefficient at the open-end of the microstrip feedline. The return loss is calculated from this factor over a frequency band of $38 \mathrm{GHz}$ and compared with measurement and found very good agreement. Radiation pattern theory is derived in such a way that the proximitycoupled microstrip antenna mounted on the back of the lens radiates into it and illuminates the interior side of the hemispherical surface. We ensure that the fields arrive at this surface are far fields so that we can apply ray tracing method in order to calculate the fields. We then calculate the fields on the exterior side of the hemisphere using appropriate transmission coefficients (parallel and perpendicular polarizations) for the interface between lens' dielectric and free space media. Equivalent surface electric and magnetic current densities on the hemispherical surface are obtained from these fields. These two current densities are incorporated into radiation integral equations and far field patterns in free space are determined. This method for determination of far field patterns is called Schelkunoff Principle or Huygens' Principle or Field Equivalence Principle. Once again we achieved good agreement between theoretical and measured radiation patterns. 
This chapter is organized as follows. Section 2 presents determination of dielectric lens geometry for which exiting rays from the lens travel in parallel with optical axis of the lens. The determined geometry is true ellipsoid lens and verified that the Snell's Law of Refraction is satisfied. Modification of true ellipsoid lens to extended hemispherical lens is presented in Section 3. Section 4 presents formulating and computing for radiation patterns of extended hemispherical dielectric lens. It comprises analysis of radiation of patch into lens by cavity model and analysis of radiation of lens into free space. Design of proximitycoupled microstrip antenna and the lens, and comparison between theoretical and measured return loss and radiation patterns are presented in Section 5. Results and discussion, and conclusion are followed there after.

\section{Determination of dielectric lens geometry}

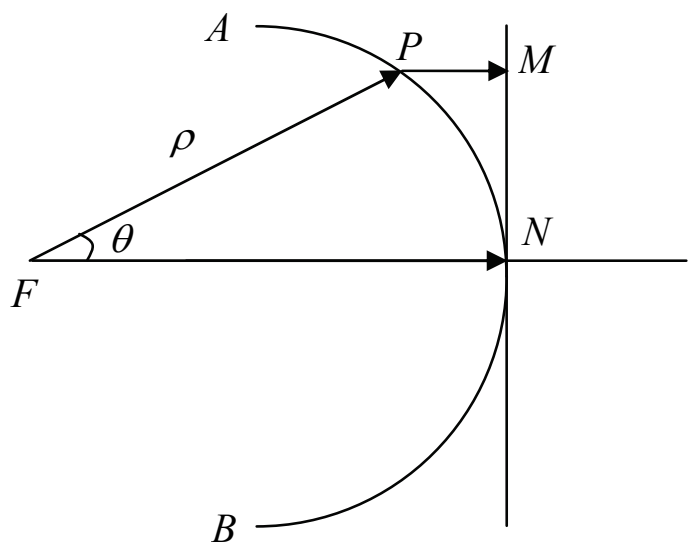

Fig. 2-1 Unknown Geometry of the dielectric lens

Since the dielectric lens' size is chosen large compared with wavelength, the far fields can be determined using ray tracing method. The rays are perpendicular to wavefronts. We now consider a dielectric lens with unknown geometry and having dielectric constant $\varepsilon_{r}$, refractive index $\eta$ and holds $\eta=\sqrt{\varepsilon_{r}}$. In Figure 2-1, ANB is unknown surface curvature of the lens under determination. $F$ is focal point and $F N$ is taken as optical axis. We place an optical source at focal point, $F$ and use spherical coordinate system $(\rho, \theta, \phi)$ for determination of required geometry of the lens while taking symmetry about $\phi . P$ is a point on the lens' surface and $M N$ is a plane perpendicular to the $F N$ axis. For a wavefront travelled a distance $x$, its phase change is $\beta x$, where wave number $\beta=\frac{2 \pi}{\lambda}$. If $\lambda_{d}$ is the wavelength in the lens' dielectric medium and $\lambda_{0}$ is the corresponding wavelength in free space, then $\lambda_{d}=\frac{\lambda_{0}}{\eta}$. We want that the optical source at $\mathrm{F}$ emits rays which are incident on lens' surface and refract into free space as parallel rays and they are also parallel to the optical axis of the lens. These parallel rays will form a wavefront on the plane $M N$ which perpendicular to the optical axis. For a plane wave, there is equal phase on each and every point on 
wavefront. Consider (1) a ray travels from point $\mathrm{F}$ to point $\mathrm{P}$ in the dielectric lens, then refracts at point $\mathrm{P}$ and travels in free space to point $\mathrm{M}$; (2) another ray travels from point $\mathrm{F}$ to point $\mathrm{N}$ along the optical axis in the dielectric lens. On arrival of, ray in (1) at point M, and ray in (2) at point $\mathrm{N}$, change of their phases must be the same in order to form a plane wave on the plane MN. A phase change equation can be written as follow for ray in (1) and ray in (2).

$$
\beta_{d}[F P]+\beta_{0}[P M]=\beta_{d}[F N]
$$

The appropriate quantities being substituted in (2-1) will yield

$$
\frac{2 \pi \eta}{\lambda_{0}} \rho+\frac{2 \pi}{\lambda_{0}}[F N-\rho \cdot \cos (\theta)]=\frac{2 \pi \eta}{\lambda_{0}}[F N]
$$

This equation can be further simplified as

$$
\rho=\frac{k}{1-e \cdot \cos (\theta)}
$$

Where

$$
k=\frac{\eta-1}{\eta}[F N]
$$

and, the eccentricity

$$
e=\frac{1}{\eta}
$$

It is obvious that $e<1$ since the refractive index $\eta>1$, and Equation (2-3) represents an ellipsoid. This finding characterizes the dielectric ellipsoid lens with $F$ as one of the two focal points and other is at the right side. The rays diagram is shown in Figure 2-2.

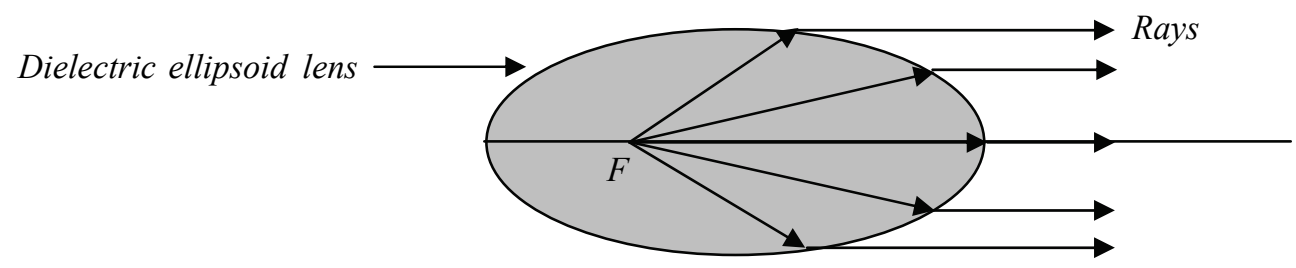

Free space

Fig. 2-2 Ray diagram of dielectric ellipsoid lens and an optical source placed at a focal point $F$.

Figure 2-2 shows an optical source placed at a focal point, F of dielectric ellipsoid lens, emanating rays to its right hand side directions. They are incident on the lens' surface, refract and exit into free space as parallel rays. We will investigate further whether this dielectric ellipsoid lens satisfies the well known Snell's Law of Refraction which states as follows.

$$
\frac{\sin \left(\psi_{i}\right)}{\sin \left(\psi_{r}\right)}=\frac{1}{\eta}
$$


$\psi_{i}$ and $\psi_{r}$ are incident and refracted angles respectively, and $\eta$ is the refractive index of the dielectric ellipsoid lens. Drawing for this investigation is shown in figure (2-3).

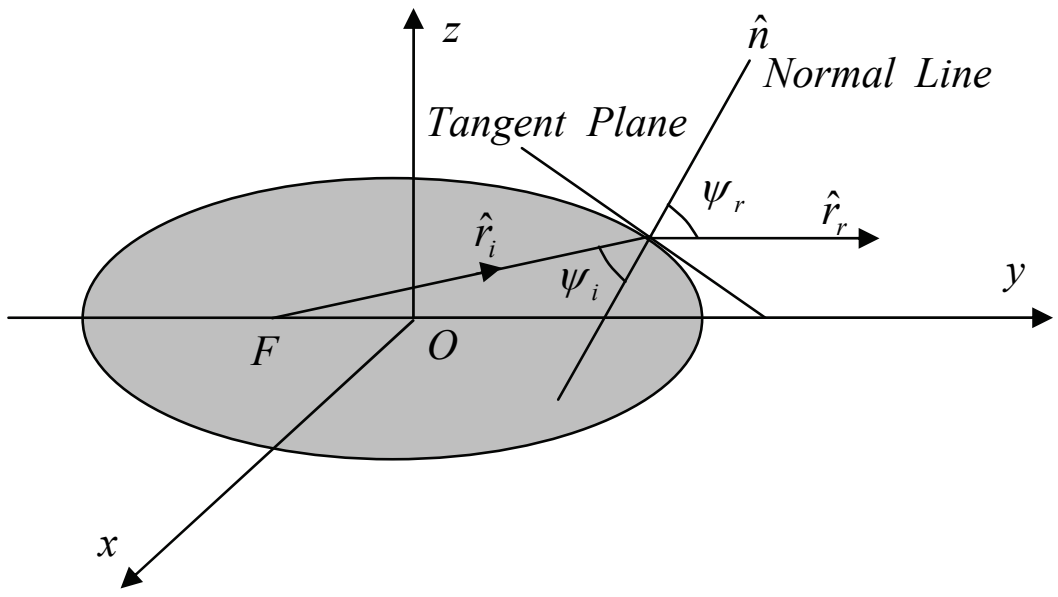

Fig. 2-3 Incident ray emitted from focal point $F$ and its refracted ray of dielectric ellipsoid lens Equation for this dielectric ellipsoid in Cartesian coordinate system and its associated can be written as,

$$
\begin{aligned}
& \frac{x^{2}+z^{2}}{b^{2}}+\frac{y^{2}}{a^{2}}=1 \\
& b^{2}=a^{2}\left(1-e^{2}\right)
\end{aligned}
$$

$a$ and $b$ are semi-major and semi-minor axes respectively. We will rewrite (2-7) as,

$$
\phi=\frac{x^{2}+z^{2}}{b^{2}}+\frac{y^{2}}{a^{2}}
$$

Then $\phi=1$ and is a constant. Using Vector Calculus, we can determine a unit vector $(\hat{n})$ along normal line on the lens surface as,

$$
\hat{n}=\frac{\vec{\nabla} \phi}{|\vec{\nabla} \phi|}=\frac{\frac{2}{b^{2}}(x \cdot \hat{i}+z \cdot \hat{k})+\frac{2}{a^{2}} y \cdot \hat{j}}{\sqrt{\left(\frac{2}{b^{2}}\right)^{2}\left(x^{2}+z^{2}\right)+\left(\frac{2}{a^{2}}\right)^{2} y^{2}}}
$$

Making use of (2-7) and (2-8) above equation can be simplified as,

$$
\hat{n}=\frac{\frac{x \cdot \hat{i}+z \cdot \hat{k}}{b^{2}}+\frac{y}{a^{2}} \cdot \hat{j}}{\sqrt{a^{2}-e^{2} \cdot y^{2}}} a b
$$


A unit vector $\left(\hat{r}_{i}\right)$, parallel to the incident ray emanating from focal point $\mathrm{F}$ can be determined as,

$$
\hat{r}_{i}=\frac{x \cdot \hat{i}+(y+a \cdot e) \cdot \hat{j}+z \cdot \hat{k}}{\sqrt{x^{2}+(y+a \cdot e)^{2}+z^{2}}}
$$

Note that distance between focal point $\mathrm{F}$ and origin $\mathrm{O}$ is a.e. Simplifying above equation using (2-7) and (2-8) yields,

$$
\hat{r}_{i}=\frac{x \cdot \hat{i}+(y+a \cdot e) \cdot \hat{j}+z \cdot \hat{k}}{a+e \cdot y}
$$

On arrival of the incident ray at a point on the tangent plane, it refracts, exits into free space as refracted ray and travels in parallel with the optical axis or y-axis. A unit vector $\left(\hat{r}_{r}\right)$ parallel to this refracted ray is,

$$
\begin{gathered}
\hat{r}_{r}=\hat{j} \\
\cos \left(\psi_{i}\right)=\hat{n} \cdot \hat{r}_{i}=\frac{\frac{x^{2}+z^{2}}{b^{2}}+\frac{y(y+a \cdot e)}{a^{2}}}{(a+e \cdot y) \cdot \sqrt{a^{2}-e^{2} \cdot y^{2}}} a b
\end{gathered}
$$

Simplifying above equation with (2-7) and (2-8) yields

$$
\begin{gathered}
\cos \left(\psi_{i}\right)=\frac{b}{\sqrt{a^{2}-e^{2} \cdot y^{2}}} \\
\sin \left(\psi_{i}\right)=\sqrt{1-\cos ^{2}\left(\psi_{i}\right)}=e \sqrt{\frac{a^{2}-y^{2}}{a^{2}-e^{2} \cdot y^{2}}} \\
\cos \left(\psi_{r}\right)=\hat{n} \cdot \hat{j}=\frac{\frac{y}{a^{2}}}{\sqrt{a^{2}-e^{2} \cdot y^{2}}} a b \\
\sin \left(\psi_{r}\right)=\sqrt{1-\cos ^{2}\left(\psi_{r}\right)}=\sqrt{\frac{a^{2}-y^{2}}{a^{2}-e^{2} \cdot y^{2}}}
\end{gathered}
$$

Then, from (2-16), (2-17) and (2-5),

$$
\frac{\sin \left(\psi_{i}\right)}{\sin \left(\psi_{r}\right)}=e=\frac{1}{\eta}
$$

We now have verified that the Snell's Law of Refraction is satisfied with (2-18) for our dielectric ellipsoid lens. 


\section{Modification of true ellipsoid lens to extended hemispherical lens}

For ease of manufacture, true ellipsoid dielectric lens is modified to extended hemispherical dielectric lens as synthesized ellipsoid. The reason for using an extended hemispherical lens is based on what can be made practically and can be synthesized to give the properties of a true ellipsoid. Producing of extended hemispherical dielectric lens, Figure 3-1, is in such a way that the surface-curvature of the hemispherical surface has the close match to that of the true ellipsoid lens. Comparison is shown in Figure 3-2, which is for two dimensions since the geometry is rotationally symmetric.

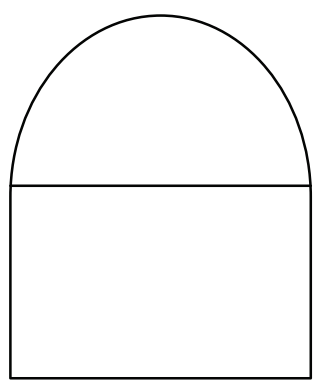

Fig. 3-1 Extended hemisphere

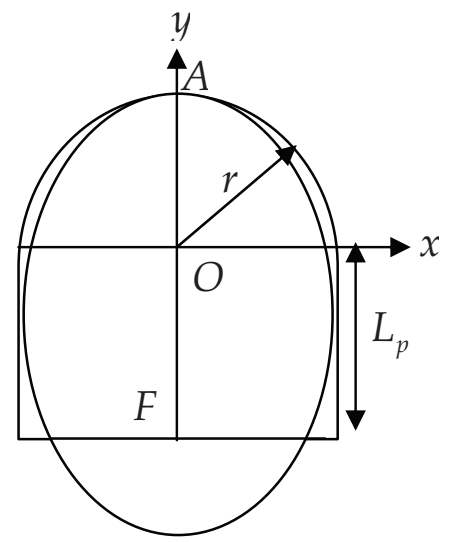

Fig. 3-2 Matching of extended hemisphere to ellipse

In Figure 3-2, $\mathrm{F}$ is the more distant focus of the ellipse from the vertex point $\mathrm{A}$. The equation for an ellipse is,

$$
\frac{x^{2}}{b^{2}}+\frac{y^{2}}{a^{2}}=1
$$

The foci are at $\pm c$ and

$$
c=\sqrt{a^{2}-b^{2}}
$$

The eccentricity $e$ is given by, 


$$
e=\frac{\sqrt{a^{2}-b^{2}}}{a}=\frac{1}{\eta}
$$

From Equation (3-3), following can be obtained,

$$
b=a \sqrt{1-\frac{1}{\eta^{2}}}
$$

From Equation (3-2) and (3-3), following can be found,

$$
c=\frac{a}{\eta}
$$

In Figure 3-2, the equation for semicircle of unit radius is (for two dimensions),

$$
x^{2}+y^{2}=1
$$

For the ellipse in Figure 3-2,

$$
\mathrm{AF}=a+c
$$

In Figure 3-2, with reference to the extended hemispherical lens,

$$
\mathrm{AF}=1+L_{p}
$$

Since Equation (3-7) and (3-8) are the same in magnitude,

$$
L_{p}=a+c-1
$$

In Figure 3-2, the ellipse has been shifted down by a value $y_{0}=L_{p}-c$ so that the focus of the ellipse has the same coordinates as that of the extended hemisphere. For $a>1$, the vertex of ellipse is $(a-1)$ unit above that of unit radius semi-circle. In order for the two vertexes overlap, the ellipse must be shifted down by $(a-1)$ unit and (3-1) will change as

$$
\frac{x^{2}}{b^{2}}+\frac{(y+a-1)^{2}}{a^{2}}=1
$$

By (3-6) a semi-circle $(y>0)$ with unit radius can be plotted. For a given refractive index, using (3-10), in combination with (3-4) for elimination of $b$, various ellipses $(y>0)$ can be traced with different parameters for $a$. We have seen that $a=1$ does not give close match between semi-circle and ellipse. For a given refractive index, $a$ only was varied until a close match was achieved between the curvature of the ellipse and that of the semi-circle with unit radius. Our selected material for the extended hemispherical dielectric lens is ultra high density polyethylene plastic with $\varepsilon_{r}=2.35$ which yields $\eta=\sqrt{\varepsilon_{r}}=1.53$. From our comparison, we are satisfied with $a=1.6$. Using (3-5) and (3-9), we received extension length of the hemisphere, with unit radius, as 1.65. This yields extension length, $L$, of hemisphere with radius $R$ using ultra high density polyethylene plastic is,

$$
L=1.65 R
$$




\section{Formulating and computing for radiation patterns}

This section documents the analysis into far field radiation patterns of extended hemispherical dielectric lens. Since the proximity-coupled microstrip patch antenna is placed at the back of the lens the radiation will illuminate the whole interior side of the lens' hemispherical surface.

\subsection{Analysis of radiation of patch into lens by cavity model}

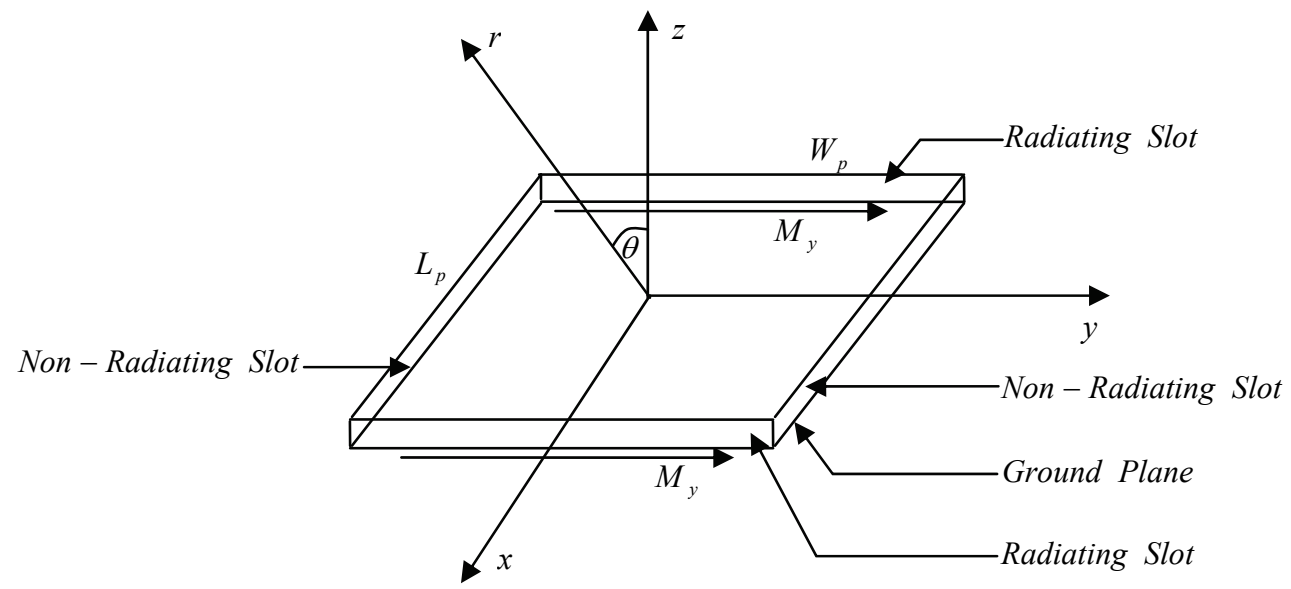

Fig. 4-1 Generation of magnetic current, $M_{y}$, on radiating slots

Figure 4-1 shows the microstrip patch in the $x-y$ plane at $z=0$ above infinitely large conducting ground plane which is located at $\mathrm{z}=-\mathrm{d}$. The length and width of the patch are $L_{p}$ and $W_{p}$ respectively. The centre of the patch is placed at the origin of the Cartesian coordinate system. It is supposed that the electric current is flowing along positive-x direction. By using the cavity model technique as shown in Section 14.2.2 of (Balanis, 1997), radiation from the patch can be derived from the two same phase and equal magnitude magnetic current sources on the radiating slots. The two magnetic current sources, $M_{y}$, are $\mathrm{y}$-directed and uniformly distributed along the slots. The process of derivation is started according to the equation (12-12c) and (12-15a) of the reference (Balanis, 1997) as follows:

$$
L_{\theta}=\int_{-d}^{0} \int_{-\frac{W_{p}}{2}}^{+\frac{W_{p}}{2}} M_{y} \cos \theta \sin \phi e^{j k\left(y^{\prime} \sin \theta \sin \phi+z^{\prime} \cos \theta\right)} d y^{\prime} d z^{\prime}
$$

this yields

$$
L_{\theta}=\cos \theta \sin \phi \frac{\sin \left\{\frac{k W_{p} \sin \theta \sin \phi}{2}\right\} \sin \left\{\frac{k d \cos \theta}{2}\right\}}{\frac{k \sin \theta \sin \phi}{2}} e^{-j \frac{k d \cos \theta}{2}}
$$


$M_{y}$ in Equation (4-1) is the y-directed uniform magnetic current and is normalized by setting it to unity in Equation (4-2). According to Equation (12-12d) and (12-15a) of reference (Balanis, 1997) the following can be derived:

$$
L_{\phi}=\int_{-d}^{0} \int_{-\frac{W_{p}}{2}}^{+\frac{W_{p}}{2}} M_{y} \cos \phi e^{j k\left(y^{\prime} \sin \theta \sin \phi+z^{\prime} \cos \theta\right)} d y^{\prime} d z^{\prime}
$$

which results

$$
L_{\phi}=\cos \phi \frac{\sin \left\{\frac{k W_{p} \sin \theta \sin \phi}{2}\right\}}{\frac{k \sin \theta \sin \phi}{2}} \frac{\sin \left\{\frac{k d \cos \theta}{2}\right\}}{\frac{k \cos \theta}{2}} e^{-j \frac{k d \cos \theta}{2}}
$$

Again, $M_{y}$ in Equation (4-3) is the y-directed uniform magnetic current and normalized by setting it to unity in Equation (4-4). The E-plane array factor (AF) is given below (Balanis, 1997),

$$
A F=2 \cos \left\{\frac{k L_{p}}{2} \sin \theta \cos \phi\right\}
$$

According to Equation (12-10b) and Section 14.2.2 of (Balanis, 1997) the following can be found:

$$
\begin{gathered}
E_{\theta}=-\frac{j k e^{-j k r}}{4 \pi r} L_{\phi}(A F) \\
E_{\phi}=\frac{j k e^{-j k r}}{4 \pi r} L_{\theta}(A F)
\end{gathered}
$$

By Equation (4-6),

$$
\begin{aligned}
E_{\theta}=-\frac{j k e^{-j k r}}{4 \pi r} \cos \phi & \frac{\sin \left\{\frac{k W_{p} \sin \theta \sin \phi}{2}\right\} \sin \left\{\frac{k d \cos \theta}{2}\right\}}{\frac{k \sin \theta \sin \phi}{2}} \frac{\frac{k \cos \theta}{2}}{2} e^{-j \frac{k d \cos \theta}{2}} \\
& \times 2 \cos \left\{\frac{k L_{p}}{2} \sin \theta \cos \phi\right\}
\end{aligned}
$$

and by Equation (4-7),

$$
\begin{gathered}
E_{\phi}=\frac{j k e^{-j k r}}{4 \pi r} \cos \theta \sin \phi \frac{\sin \left\{\frac{k W_{p} \sin \theta \sin \phi}{2}\right\}}{\frac{k \sin \theta \sin \phi}{2}} \frac{\sin \left\{\frac{k d \cos \theta}{2}\right\}}{\frac{k \cos \theta}{2}} e^{-j \frac{k d \cos \theta}{2}} \\
\times 2 \cos \left\{\frac{k L_{p}}{2} \sin \theta \cos \phi\right\}
\end{gathered}
$$




\subsection{Analysis of radiation of lens into free space}

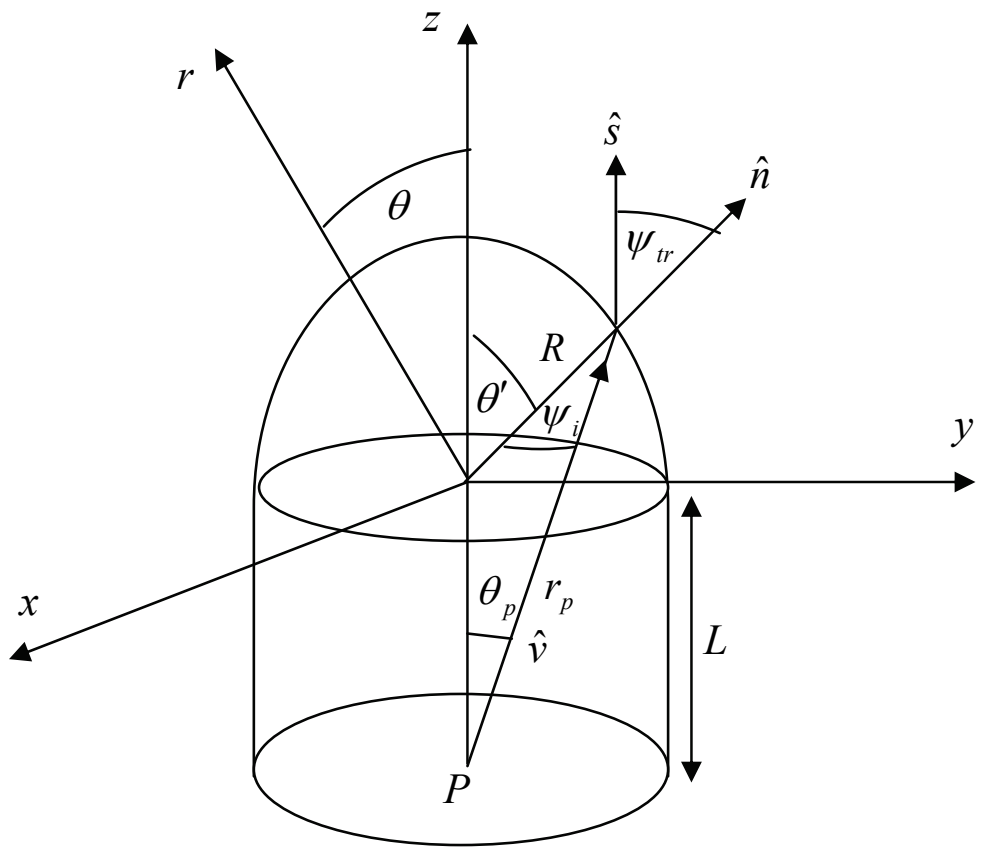

Fig. 4-2 Lens geometry and rays diagram

In Figure 4-2, the intersection point of the $x, y$ and z-axis is the origin $(0,0,0)$ of the Cartesian coordinate system. $\mathrm{R}$ is the radius of the hemisphere and $\mathrm{L}$ is the extension length. The point $\mathrm{P}$ is the centre of the patch which is lying in the $\mathrm{x}-\mathrm{y}$ plane at $\mathrm{z}=-\mathrm{L}$. The patch orientation is the same as in Figure 4-1 and the electric current flowing on the patch is along positive-x direction. For transformations of Equation (4-8) and (4-9) to Figure 4-2, $r, \theta$ and $\phi$ will change to $r_{p}, \theta_{p}$ and $\phi_{p}$ respectively. The $\left(r_{p}, \theta_{p}, \phi_{p}\right)$ coordinates have their origin at the point P. Next transformation is performed from $\left(r_{p}, \theta_{p}, \phi_{p}\right)$ to $\left(R, \theta^{\prime}, \phi^{\prime}\right)$, whose origin is at the intersections of $\mathrm{x}, \mathrm{y}, \mathrm{z}$ axes and $R$ is constant.

$R$ and $\mathrm{L}$ are known constant parameters of the lens, and so for a given $\theta^{\prime}, \theta_{p}$ can be found. Equation (4-8) and (4-9) can be expressed in terms of $\left(R, \theta^{\prime}, \phi^{\prime}\right)$ as follows:

$$
\begin{aligned}
& E_{\theta p}=E_{\theta p}\left(R, \theta^{\prime}, \phi^{\prime}\right) \\
& E_{\phi p}=E_{\phi p}\left(R, \theta^{\prime}, \phi^{\prime}\right)
\end{aligned}
$$

Next the unit vectors shown on Figure 4-2 are to be determined. The surface normal unit vector is expressed as follows:

$$
\hat{n}=\sin \theta^{\prime} \cos \phi^{\prime} \hat{x}+\sin \theta^{\prime} \sin \phi^{\prime} \hat{y}+\cos \theta^{\prime} \hat{z}
$$

The ray path inside the lens is, 


$$
\vec{V}=R \sin \theta^{\prime} \cos \phi^{\prime} \hat{x}+R \sin \theta^{\prime} \sin \phi^{\prime} \hat{y}+\left(R \cos \theta^{\prime}+L\right) \hat{z}
$$

Its unit vector is,

$$
\hat{v}=\frac{\vec{V}}{|\vec{V}|}
$$

The vector that defines the component of electric field in the perpendicular plane of incident ray can be found as:

$$
\vec{P}_{\perp}=\hat{n} \times \hat{v}
$$

where its unit vector is:

$$
\hat{p}_{\perp}=\frac{\vec{P}_{\perp}}{\left|\vec{P}_{\perp}\right|}
$$

The unit vector that defines the component of the electric field in the parallel plane of incident ray can be found as:

$$
\hat{p}_{||}=\hat{p}_{\perp} \times \hat{v}
$$

The parallel polarization transmission coefficient and perpendicular polarization transmission coefficient can be found respectively based on Section 2-8 of (Collin, 1966):

$$
\begin{gathered}
T_{||}=\frac{2 \eta \cos \psi_{i}}{\cos \psi_{i}+\eta \sqrt{1-\eta^{2} \sin ^{2} \psi_{i}}} \\
T_{\perp}=\frac{2 \eta \cos \psi_{i}}{\eta \cos \psi_{i}+\sqrt{1-\eta^{2} \sin ^{2} \psi_{i}}}
\end{gathered}
$$

Where $\eta$ is the refractive index of the material of lens and $\psi_{i}$ is the ray incident angle as shown in the Figure 4-2. Equation (4-18) and (4-19) are valid for $0 \leq \psi_{i} \leq \psi_{i c}$ where $\psi_{i c}$ holds as:

$$
1-\eta^{2} \sin ^{2} \psi_{i c}=0
$$

or

$$
\psi_{i c}=\arcsin \left(\frac{1}{\eta}\right)
$$

$\psi_{i c}$ is called the critical angle. When $\psi_{i}>\psi_{i c}$, the right hand sides of Equation (4-18) and (419) are not valid. All the incident rays reflect back into the lens and so, this phenomenon is called the total internal reflection. In such a situation, the left hand side of Equation (4-18) and (4-19) are zero, $T_{||}=T_{\perp}=0$.

From Figure 4-2, it can be seen that

$$
\psi_{i}=\theta^{\prime}-\theta_{p}
$$


$\theta_{p}$ can be found, using elementary trigonometry, and substituted in (4-21)

$$
\psi_{i}=\theta^{\prime}-\arcsin \left[\frac{R \sin \theta^{\prime}}{\sqrt{R^{2}+L^{2}+2 R L \cos \theta^{\prime}}}\right]
$$

From Equation (4-22), it is noticed that the variable $\psi_{i}$ is only a function of the variable $\theta^{\prime}$. The refracted angle, $\psi_{t r}$, can be found by Snell's Law as,

$$
\frac{\sin \left(\psi_{i}\right)}{\sin \left(\psi_{t r}\right)}=\frac{1}{\eta}
$$

or

$$
\psi_{t r}=\arcsin \left[\eta \sin \left(\psi_{i}\right)\right]
$$

Equation (4-22) is substituted into Equation (4-23) to express the variable $\psi_{t r}$ in term of the variable $\theta^{\prime}$ :

$$
\psi_{t r}=\arcsin \left[\eta \sin \left(\theta^{\prime}-\arcsin \left\{\frac{R \sin \theta^{\prime}}{\sqrt{R^{2}+L^{2}+2 R L \cos \theta^{\prime}}}\right\}\right)\right]
$$

The components of the transmitted electric field at the exterior side of the hemispherical surface of the lens have been presented in (Filipovic et al., 1997) and they are given as follows:

$$
\begin{aligned}
& E_{x}=\left(-E_{\phi p}\right) T_{\perp}\left(\hat{x} \cdot \hat{p}_{\perp}\right)+\left(-E_{\theta p}\right) T_{||}\left[\left(\hat{x} \cdot \hat{p}_{||}\right) \cos \left(\psi_{t r}-\psi_{i}\right)-(\hat{x} \cdot \hat{v}) \sin \left(\psi_{t r}-\psi_{i}\right)\right] \\
& E_{y}=\left(-E_{\phi p}\right) T_{\perp}\left(\hat{y} \cdot \hat{p}_{\perp}\right)+\left(-E_{\theta p}\right) T_{||}\left[\left(\hat{y} \cdot \hat{p}_{||}\right) \cos \left(\psi_{t r}-\psi_{i}\right)-(\hat{y} \cdot \hat{v}) \sin \left(\psi_{t r}-\psi_{i}\right)\right] \\
& E_{z}=\left(-E_{\phi p}\right) T_{\perp}\left(\hat{z} \cdot \hat{p}_{\perp}\right)+\left(-E_{\theta p}\right) T_{||}\left[\left(\hat{z} \cdot \hat{p}_{||}\right) \cos \left(\psi_{t r}-\psi_{i}\right)-(\hat{z} \cdot \hat{v}) \sin \left(\psi_{t r}-\psi_{i}\right)\right]
\end{aligned}
$$

Where $E_{\theta p}$ and $E_{\phi p}$ are given in Equation (4-10) and (4-11) respectively, based on Equation (4-8) and (4-9). The resultant transmitted electric field at the exterior side of the hemispherical surface of the lens is:

$$
\vec{E}=E_{x} \hat{x}+E_{y} \hat{y}+E_{z} \hat{z}
$$

The ray path unit vector outside the lens can be written as follows:

$$
\hat{s}=s_{x} \hat{x}+s_{y} \hat{y}+s_{z} \hat{z}
$$

The components have already been given in (Filipovic et al., 1997) and they are shown as follows:

$$
\begin{aligned}
& s_{x}=v_{x} \cos \left(\psi_{t r}-\psi_{i}\right)+\left(\hat{x} \cdot \hat{p}_{||}\right) \sin \left(\psi_{t r}-\psi_{i}\right) \\
& s_{y}=v_{y} \cos \left(\psi_{t r}-\psi_{i}\right)+\left(\hat{y} \cdot \hat{p}_{||}\right) \sin \left(\psi_{t r}-\psi_{i}\right)
\end{aligned}
$$




$$
s_{z}=v_{z} \cos \left(\psi_{t r}-\psi_{i}\right)+\left(\hat{z} \cdot \hat{p}_{||}\right) \sin \left(\psi_{t r}-\psi_{i}\right)
$$

The normalized resultant magnetic field at the exterior side of the hemispherical surface of the lens is:

$$
\vec{H}=\hat{s} \times \vec{E}
$$

The equivalent surface electric current density and surface magnetic current density on the exterior side of the hemispherical surface of the lens are:

$$
\begin{aligned}
\vec{J}_{s} & =\hat{n} \times \vec{H} \\
\vec{M}_{s} & =-\hat{n} \times \vec{E}
\end{aligned}
$$

By Schelkunoff's principle (Balanis, 1997), Equation (4-34) and (4-35) are incorporated into the radiation integral equations and far fields of the lens are determined.

$$
\begin{gathered}
N_{\theta}=\iint_{S}\left[J_{x} \cos \theta \cos \phi+J_{y} \cos \theta \sin \phi-J_{z} \sin \theta\right] e^{+j k R \cos \psi} d s^{\prime} \\
N_{\phi}=\iint_{S}\left[-J_{x} \sin \phi+J_{y} \cos \phi\right] e^{+j k R \cos \psi} d s^{\prime} \\
L_{\theta}=\iint_{S}\left[M_{x} \cos \theta \cos \phi+M_{y} \cos \theta \sin \phi-M_{z} \sin \theta\right] e^{+j k R \cos \psi} d s^{\prime} \\
L_{\phi}=\iint_{S}\left[-M_{x} \sin \phi+M_{y} \cos \phi\right] e^{+j k R \cos \psi} d s^{\prime}
\end{gathered}
$$

$\cos \psi$ in Equation (4-36), (4-37), (4-38) and (4-39) is

$$
\cos \psi=\hat{a}_{r} \cdot \hat{n}
$$

$\hat{a}_{r}$ in Equation (4-40) is expressed as,

$$
\hat{a}_{r}=\sin \theta \cos \phi \hat{x}+\sin \theta \sin \phi \hat{y}+\cos \theta \hat{z}
$$

$\hat{n}$ was defined in Equation (4-12). Using Equation (4-41) and (4-12) in (4-40), $\cos \psi$ can be determined. $d s^{\prime}$ is the differential area element on the hemispherical surface of the lens and can be expressed in the polar coordinate form as,

$$
d s^{\prime}=R^{2} \sin \theta^{\prime} d \theta^{\prime} d \phi^{\prime}
$$

Substituting Equation (4-40) and (4-42) into Equation (4-36), (4-37), (4-38) and (4-39) allows the solution to be determined by means of solving the double integrals numerically since all the integrands are functions of the variables, $\theta^{\prime}$ and $\phi^{\prime}$. The integration limits are from 0 to $2 \pi$ for $\phi^{\prime}$ and 0 to $\frac{\pi}{2}$ for $\theta^{\prime}$. The normalized far field can be expressed as:

$$
E_{\theta}=-\left(L_{\phi}+Z_{0} N_{\theta}\right)
$$




$$
E_{\phi}=\left(L_{\theta}-Z_{0} N_{\phi}\right)
$$

where $Z_{0}$ is intrinsic impedance of free space. Since the patch current is $\mathrm{x}$-directed, varying $\theta$ while $\phi$ is kept constant at 0 degree or 90 degrees will yield E-plane or H-plane pattern respectively.

\section{Design of proximity-coupled microstrip antenna and the lens}

The configuration of cross-sectional view of proximity-coupled microstrip antenna on extended hemispherical dielectric lens is shown in figure 5-1. $h$ and $(d-h)$ are the thickness of the feedline-substrate and patch-substrate. $L_{O S}$ is the offset of the feedline from below the center of the patch. $R_{L}$ and $L_{L}$ are radius of hemisphere and extension length respectively. Using $\mathrm{C}++$ programming language on Linux operating system, we developed a design software tool in moment method for a proximity-coupled microstrip antenna residing in dielectric half space. The derived Green's functions in the code are for electric current sources residing in homogeneous dielectric medium. Since the details were given in (Pozar and Voda, 1987) and (Mall, 2008) we will not repeat here for the sake of brevity. We simulated our code for design of antenna, operating at $38 \mathrm{GHz}$, and obtained the parameters. Two $0.127 \mathrm{~mm}$ thick RT/Duroid 5870 substrates $\left(\varepsilon_{r}=2.33\right)$ were used for both patch and feedline layers. The patch dimensions were $2.175 \mathrm{~mm} \times 2.000 \mathrm{~mm}$ and the open circuit termination was $0.1 \mathrm{~mm}$ from below the centre of the patch. To couple power to and from the antenna, a K-type connector was mounted through the ground-plane and soldered to the microstrip feedline $15 \mathrm{~mm}$ below from the centre of the microstrip patch. The track with of microstripline for $50 \Omega$ was $0.33 \mathrm{~mm}$. As ultra high density polyethylene plastic material $\left(\varepsilon_{r}=2.35\right)$ was used and manufactured extended hemispherical dielectric lens, the

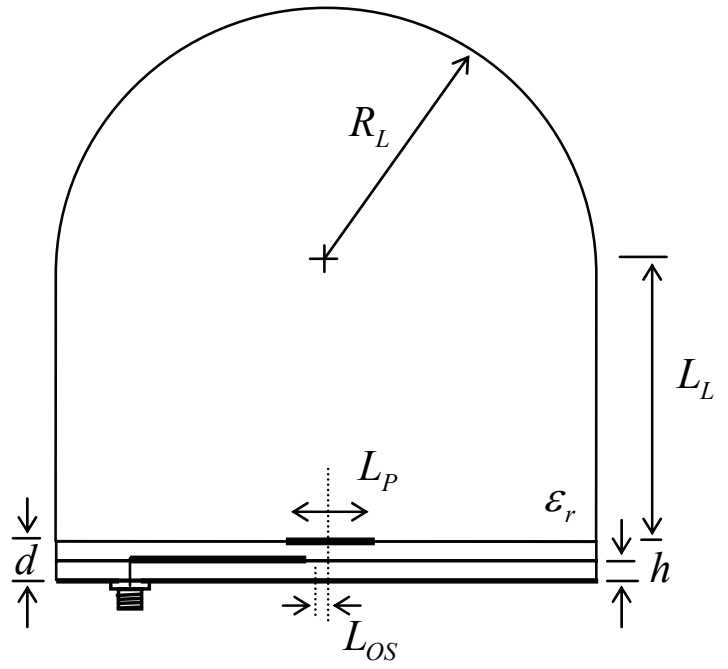

Fig. 5-1 Cross-sectional view of proximity-coupled microstrip antenna on extended hemispherical dielectric lens 
wavelength in the lens at $38 \mathrm{GHz}$ is $5.16 \mathrm{~mm}$. Our selection of hemisphere's radius as $50 \mathrm{~mm}$ is quite good for the requirement that the lens' radius is much larger than the wavelength. According to (3-11), extension length has to be $82.5 \mathrm{~mm}$.

Figure 5-2 shows the theoretical and measured return loss of the proximity-coupled lens antenna. The measurements were performed using a Wiltron 360B $40 \mathrm{GHz}$ Vector Network Analyzer. As can be seen from these results, good agreement between theory and experiment was achieved. The predicted and measured $10 \mathrm{~dB}$ return loss bandwidths are $8.0 \%$ and $8.2 \%$ respectively.

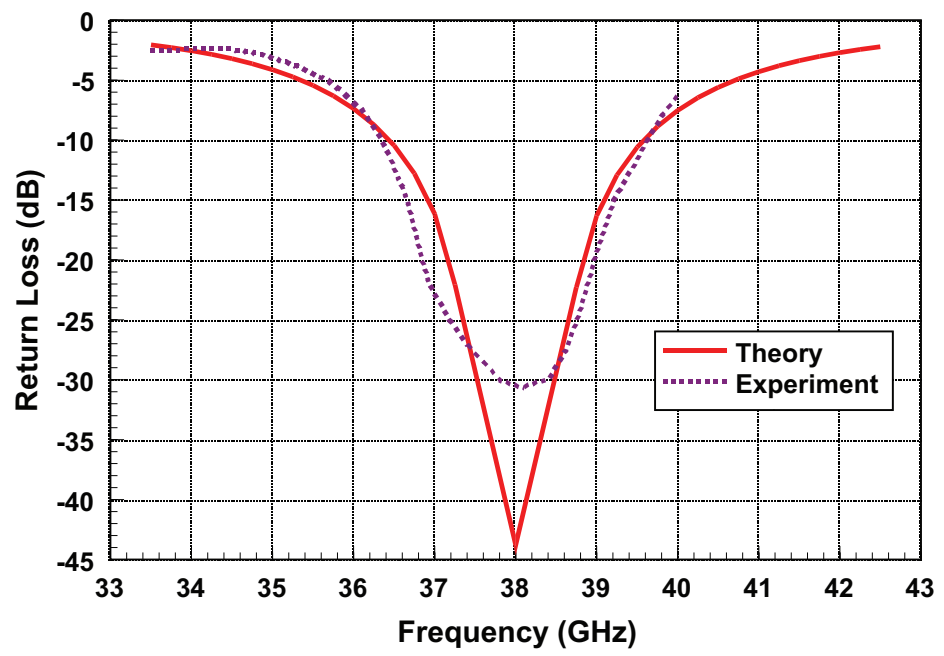

Fig. 5-2 Predicted and measured return loss curves

The predicted and measured $E$-plane and $H$-plane copolar radiation patterns at $38 \mathrm{GHz}$ are shown in Figure 5-3 and 5-4 respectively. Once again, good agreement between theory and experiment is evident from these plots, particularly in the main lobes.

\subsection{Results and discussion}

Figure 5-2 shows the predicted and measured return loss of the proximity-coupled lens antenna. The measurements were done using a Wiltron 360B $40 \mathrm{GHz}$ Vector Network Analyzer. As can be seen from these results, good agreement between theory and experiment was achieved. A high degree of precision is required in the manufacture to achieve such a good match between measured and simulated results. The predicted and measured $10 \mathrm{~dB}$ return loss bandwidths are $8.0 \%$ and $8.2 \%$, respectively. The slight shift in resonant frequency of $0.3 \%$ can be attributed to uncertainties in the dielectric properties of the materials $\left(\Delta \varepsilon_{\mathrm{r}} \pm 0.02\right)$ or even slight misalignment of the layers.

The predicted and measured E-plane and $\mathrm{H}$-plane co-polar radiation patterns at 38 GHz are shown in Figure 5-3 and 5-4, respectively. Once again good agreement between theory and experiment is evident from these plots, particularly in the main lobes. The slight discrepancy in the sidelobe level is due to total internal reflection in the dielectric lens. These reflected rays leaving opposite wall and enforce sidelobe levels. It is interesting to note that the measured first sidelobes in both planes are less in magnitude than the predicted values, 


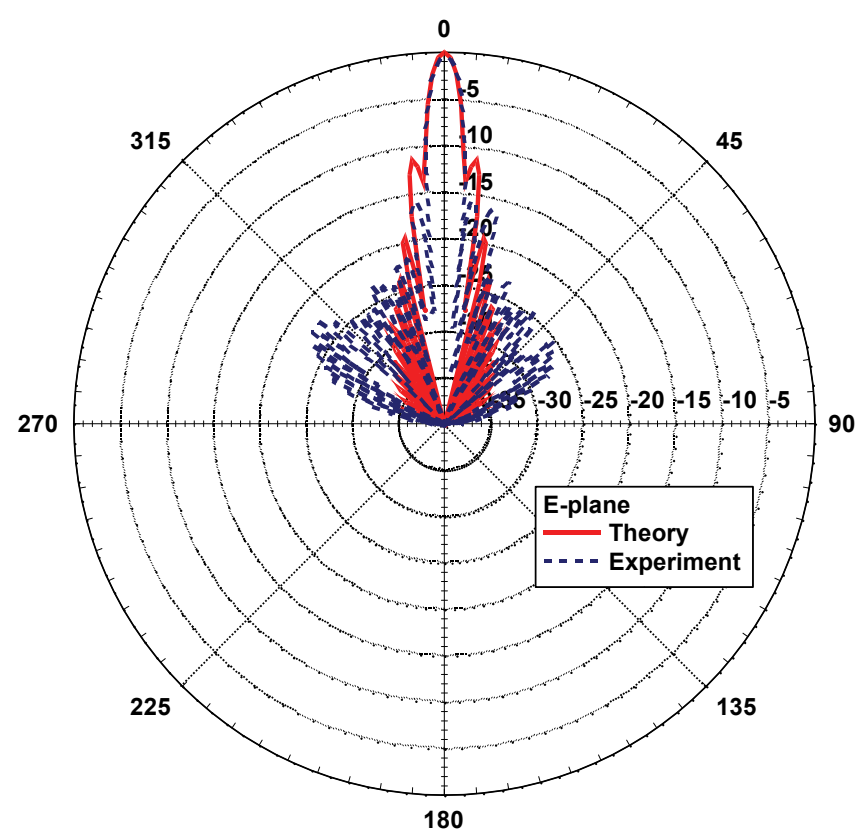

Fig. 5-3 Predicted and measured E-plane pattern at $38 \mathrm{GHz}$

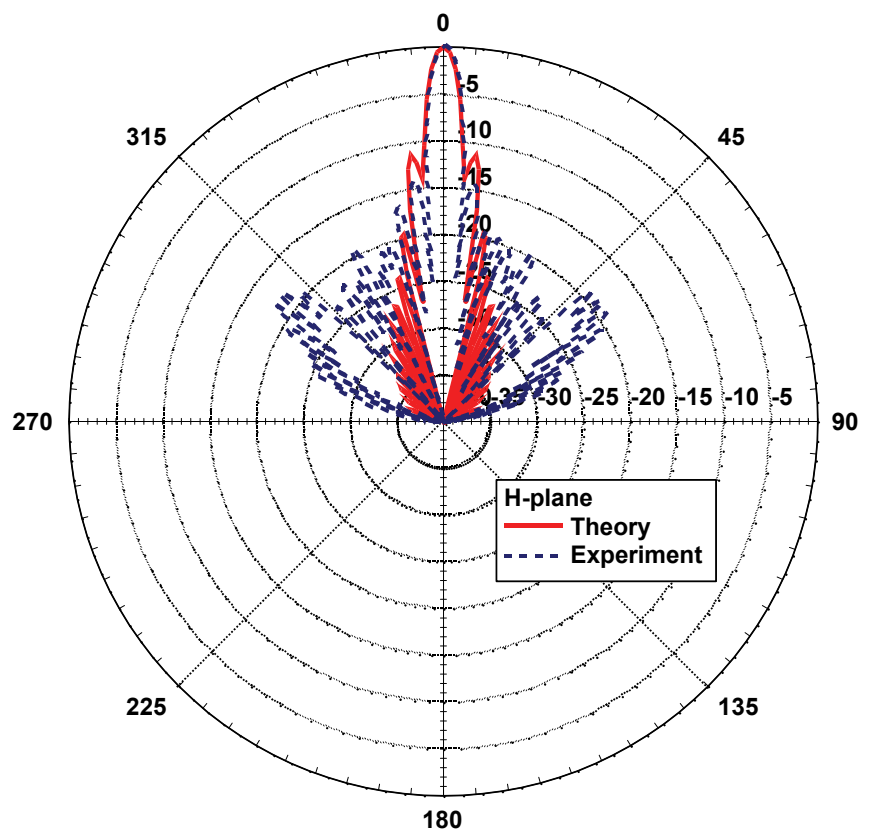

Fig. 5-4 Predicted and measured H-plane pattern at $38 \mathrm{GHz}$ 


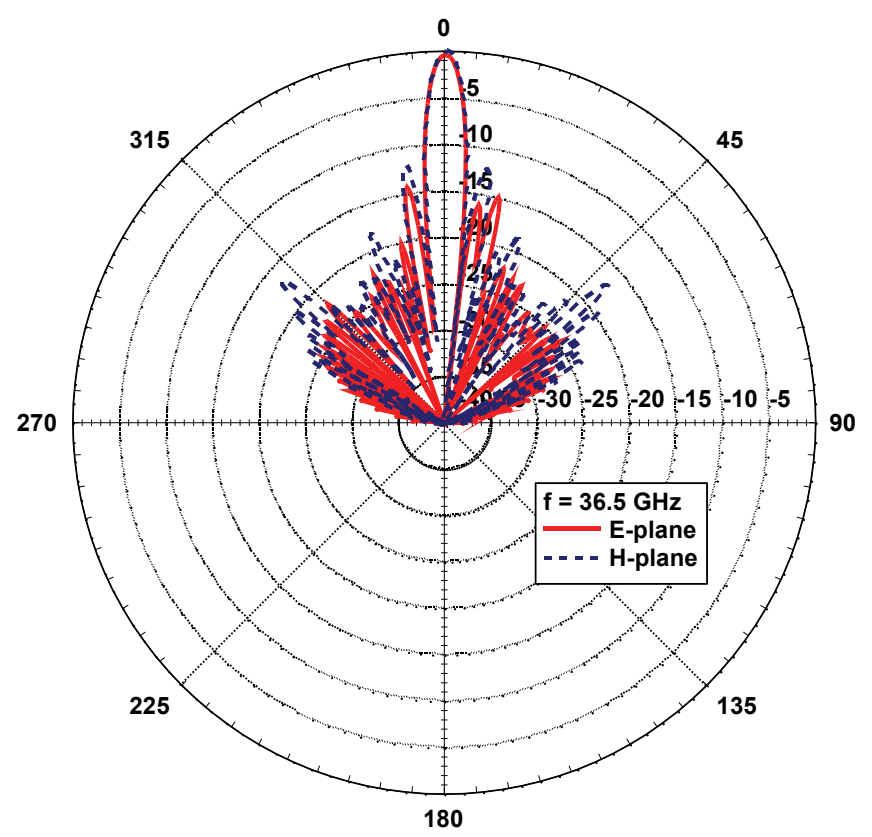

Fig. 5-5 Measured E-plane and H-plane pattern at 36.5 GHz

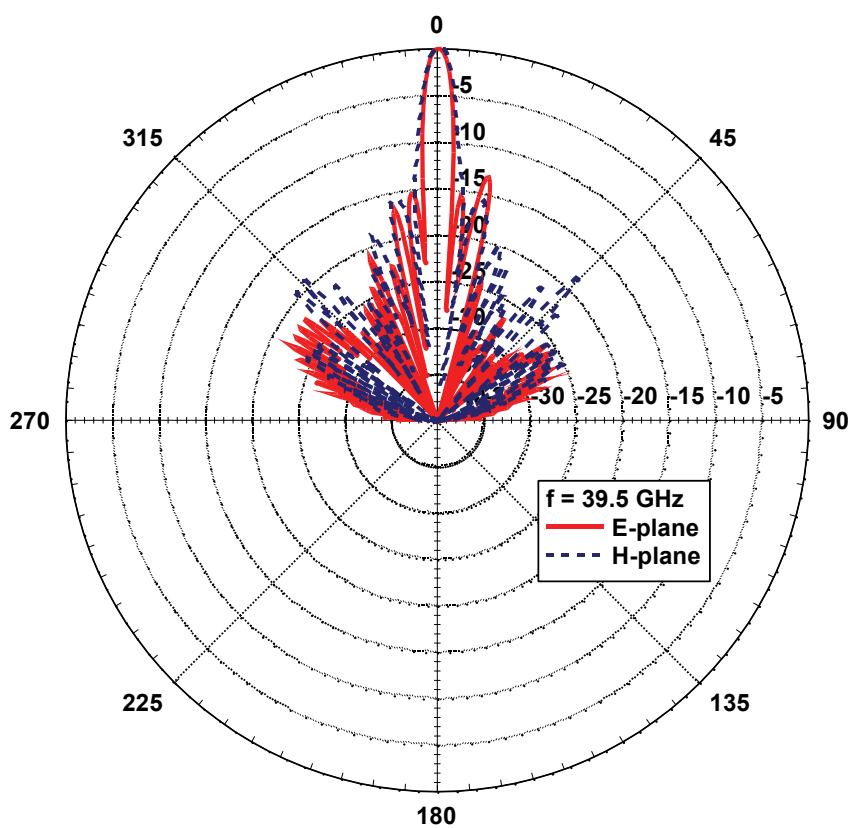

Fig. 5-6 Measured E-plane and H-plane patterns at $39.5 \mathrm{GHz}$ 
at the expense of higher order sidelobes. The $3 \mathrm{~dB}$ beamwidth is $4.5^{\circ}$ in the E-plane and $4.5^{\circ}$ in the H-plane. Directivity is computed using numerical integration method and found to be $30.2 \mathrm{~dB}$. The measured cross-polarization levels in both the E- and H-planes were $20 \mathrm{~dB}$ below the co-polar levels.

Figure 5-5 and 5-6 show the measured radiation performance of the proximity-coupled lens antenna at the band-edges of the operating frequency range. As can be seen from these plots, the radiation patterns remain relatively constant over this bandwidth. The $3 \mathrm{~dB}$ beamwidths in both the E- and $\mathrm{H}$-planes are $4.8^{\circ}$ at $36.5 \mathrm{GHz}$ and $4.2^{\circ}$ at $39.5 \mathrm{GHz}$. For the proximity-coupled patch lens-antenna, a slight asymmetry in the E-plane patterns due to spurious radiation from the feed-line, which is not taken into consideration in the numerical model, could be expected. However, as is evident from Figure 5-3, 5-4, 5-5 and 5-6 the measured patterns appear to be quite symmetrical. This indicates that the spurious radiation from these sources is minimal. Once again the cross-polarized levels were greater than $20 \mathrm{~dB}$ below the co-polarized fields for both frequencies in both planes. The front-toback ratio of the antenna, as measured, is greater than $60 \mathrm{~dB}$ over this frequency range.

\section{Conclusion}

This chapter presented analysis into proximity-coupled microstrip antenna on dielectric lens for the characteristics with high gain and pencil beam. In order to achieve this type of dielectric lens we showed theoretical determination of lens geometry for which exiting rays from the lens travel in parallel with optical axis of the lens. The determined geometry is found true ellipsoid lens and verified that the Snell's Law of Refraction is satisfied. Modification of true ellipsoid lens to extended hemispherical lens is presented. We later presented formulation and computation for radiation patterns of extended hemispherical dielectric lens. It comprises the analysis of radiation of patch into lens by cavity model and the analysis of radiation of lens into free space. After design of the proximity-coupled microstrip antenna and the lens, we presented our achievement of good agreements between theoretical and measured results over return loss and radiation patterns.

\section{References}

Filipovic, D.F.; Gearhart, S.S. and Rebeiz, G.M. (1993). Double-Slot Antennas on Extended Hemispherical and Elliptical Silicon Dielectric Lenses. IEEE Trans. on Microwave Theory \& Techniques, Vol. 41, No. 10, pp. 1738-1749.

Eleftheriades, G.V.; Brand, Y.; Zurcher, J-F and Mosig, J.R. (1997). ALPSS: A millimeterwave aperture-coupled patch antenna on a substrate lens. Electronics Letters, Vol. 33, No. 3, pp. 169-170, 30th January.

Balanis, C.A. (1997). Antenna Theory: Analysis and Design, John Wiley \& Sons, Inc.

Collin, R.E. (1966). Foundations for Microwave Engineering, McGraw-Hill Inc.

Filipovic, D.F.; Gauthier, G.P., Raman, S. and Rebeiz, G.M. (1997). Off-Axis Properties of Silicon and Quartz Dielectric Lens Antennas. IEEE Trans. on Antennas and Propagation, Vol. 45, No. 5, pp. 760-766.

Pozar, D. M. and Voda, S.M. (1987). A Rigorous Analysis of Microstripline Fed Patch Antenna. IEEE Trans. on Antennas and Propagation, Vol. 35, No. 12, pp. 1343-1349.

Mall, L. (2008). Improved Formulation for Investigation of Proximity-Coupled Microstrip Antenna in Homogeneous Dielectric Half Space. IEEE Trans. on Antennas and Propagation, Vol. 56, No. 4 pp. 927-932 


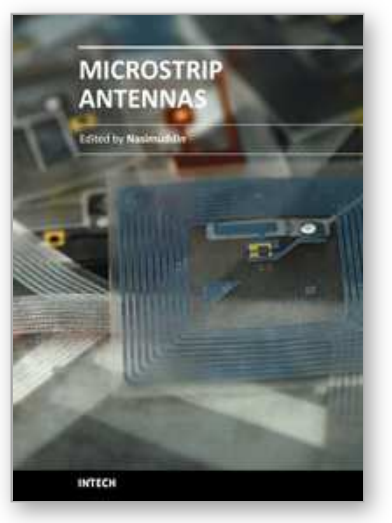

\author{
Microstrip Antennas \\ Edited by Prof. Nasimuddin Nasimuddin
}

ISBN 978-953-307-247-0

Hard cover, 540 pages

Publisher InTech

Published online 04, April, 2011

Published in print edition April, 2011

In the last 40 years, the microstrip antenna has been developed for many communication systems such as radars, sensors, wireless, satellite, broadcasting, ultra-wideband, radio frequency identifications (RFIDs), reader devices etc. The progress in modern wireless communication systems has dramatically increased the demand for microstrip antennas. In this book some recent advances in microstrip antennas are presented.

\title{
How to reference
}

In order to correctly reference this scholarly work, feel free to copy and paste the following:

Lawrence Mall (2011). Analysis into Proximity-Coupled Microstrip Antenna on Dielectric Lens, Microstrip Antennas, Prof. Nasimuddin Nasimuddin (Ed.), ISBN: 978-953-307-247-0, InTech, Available from: http://www.intechopen.com/books/microstrip-antennas/analysis-into-proximity-coupled-microstrip-antenna-ondielectric-lens

\section{INTECH}

open science | open minds

\author{
InTech Europe \\ University Campus STeP Ri \\ Slavka Krautzeka 83/A \\ 51000 Rijeka, Croatia \\ Phone: +385 (51) 770447 \\ Fax: +385 (51) 686166 \\ www.intechopen.com
}

\section{InTech China}

Unit 405, Office Block, Hotel Equatorial Shanghai

No.65, Yan An Road (West), Shanghai, 200040, China

中国上海市延安西路65号上海国际贵都大饭店办公楼405单元

Phone: $+86-21-62489820$

Fax: +86-21-62489821 
(C) 2011 The Author(s). Licensee IntechOpen. This chapter is distributed under the terms of the Creative Commons Attribution-NonCommercialShareAlike-3.0 License, which permits use, distribution and reproduction for non-commercial purposes, provided the original is properly cited and derivative works building on this content are distributed under the same license. 